\title{
MODELING OF TRANSDUCER CALIBRATION FOR PRESSURE MEASUREMENT IN NANOSECOND LASER ABLATION
}

\author{
A.A. SAMOKHIN*, P.A. PIVOVAROV, A.L. GALKIN \\ Prokhorov General Physics Institute of the Russian Academy of Sciences \\ 119991, Moscow, Vavilov str., 38 \\ *Corresponding author. E-mail: asam40@mail.ru
}

\section{DOI:10.20948/mathmontis-2020-48-6}

Summary. Pressure transducer calibration is modeled in the regime where calculated thermoacoustic signal and experimental information about normal boiling temperature attainment due to nanosecond laser pulses irradiation are used. It is shown that the regime demonstrated recently for $30 \mathrm{~ns}$ laser action is not straightforwardly applicable for shorter pulses due to strong thermoacoustic signal enhancement compared with vaporization signal near normal boiling point. For subnanosecond laser irradiation two pulses action is suggested where shorter and longer pulses are used simultaneously.

\section{INTRODUCTION.}

In [1], a method for calibrating a pressure transducer using a change in the thermoacoustic signal due to the appearance of evaporative pressure as a marker for reaching the target normal boiling point was proposed and implemented. Additional information about the actual temperature of the irradiated target with its known thermophysical parameters allows us to accurately calculate the value of thermoacoustic pressure and thereby calibrate the acoustic recording scheme used.

The fact of simultaneous recording of thermoacoustic and evaporative signals has been recorded in irradiated dielectrics, metals and semiconductors for a long time [2-5]. However, the use of this fact for the calibration of piezoelectric sensors has not been reported until recently, although piezoelectric sensors have been used in the diagnosis of pulsed exposure for more than half a century [6].

A $7 \mathrm{~mm}$ thick piezoelectric sensor operating in the current source mode was calibrated in [1] under the influence of a laser pulse $(25 \mathrm{~ns}, 1.06 \mu \mathrm{m})$ on the surface of a metal target made of liquid mercury, which was in contact with the sensor through a $3 \mathrm{~mm}$ thick glass layer. The value of thermoacoustic pressure at the time the boiling point was reached was comparable to atmospheric pressure, which facilitated its simultaneous observation with the evaporative signal.

Since the value of the thermoacoustic signal at a fixed pulse intensity grows inversely with its duration, the question arises of how much this calibration technique can be used for shorter pulses when, against the background of increased thermoacoustic pressure, the evaporation signal in the temperature range close to normal boiling becomes relatively less noticeable.

In this paper, we analyze the possibility of extending this method to shorter laser pulses.

\section{THERMOACOUSTIC PRESSURE SIGNAL IN LINEAR APPROXIMATION.}

The one-dimensional hydrodynamic problem for calculating the thermoacoustic pressure pulse when the absorbing target is heated by a laser pulse is described by the following system of equations:

$$
\frac{\partial \rho}{\partial t}+u \frac{\partial \rho}{\partial z}+\rho \frac{\partial u}{\partial z}=0
$$

2010 Mathematics Subject Classification: 78A60, 80A20, 81T80.

Key words and Phrases: Laser Ablation, Thermoacoustic Signal, Pressure Sensor Calibration, Mercury. 


$$
\begin{gathered}
\frac{\partial u}{\partial t}+u \frac{\partial u}{\partial z}+\frac{1}{\rho} \frac{\partial P}{\partial z}=0 \\
\rho C_{p}\left(\frac{\partial T}{\partial t}+u \frac{\partial T}{\partial z}\right)=\frac{\partial}{\partial z} \kappa \frac{\partial T}{\partial z}+Q \\
Q(z, t)=\alpha I(t) e^{-\alpha z} \\
I(t)=I_{m} e^{-b\left(\frac{t}{\tau_{p}}-2.5\right)^{2}}, b=2.772
\end{gathered}
$$

Here $\rho, u, T, P$ - density, speed, temperature, pressure are space-time dependent functions $(z, t) . C_{p}, \kappa$ - heat capacity and thermal conductivity (known temperature functions). $Q, \alpha, I_{m}$, $\tau_{p^{-}}$source power density, absorption coefficient, peak laser intensity and duration of a laser pulse of a Gaussian shape.

The heat equation (3) should be supplemented with initial and boundary conditions:

$$
T(z, 0)=T_{o}, \quad \kappa \frac{\partial T}{\partial z}(0, t)=0, \quad T(L, t)=T_{o}
$$

The right-hand boundary condition (6) should be set at a distance $L$, where the temperature change can be considered negligible in comparison with the quantities of interest to us, which thereby virtually cease to depend on $L$.

Let us now consider various approximate approaches to the solutions of these equations, supplemented by the equation of state $\rho(T, P)$, which are used to determine pressure pulses excited in absorbing condensed media under the action of laser radiation.

If we do not take into account the dependence of density on pressure $P$, which is true for fairly slow processes, then from (2) in the linear approximation we obtain the well-known expression for thermoacoustic pressure in terms of time derivatives of surface temperature and absorbed intensity [3]:

$$
P=-\rho \int_{0}^{x} \frac{\partial u_{l i n}}{\partial t} d x_{1} \approx \frac{1}{C_{p}} \frac{\partial \rho}{\rho \partial T}\left[\kappa \frac{\partial T}{\partial t}+\frac{\dot{I}}{\alpha}\right]
$$

It is seen from (7) that, in the case of a fixed intensity maximum, the pressure increases inversely with the duration of the laser pulse. For small values of the absorption coefficient in (7), the second term related to the volume character of the absorption is dominant, and in the opposite limiting case, the main role is played by the mechanism of diffusion thermal heating of the target. Such an explicit mechanism separation is possible only in the linear approximation (7).

These expressions are valid at distances exceeding the total heating length, but small in comparison with the length of the corresponding sound wave. Taking into account the dependence of density on pressure in a linear approximation for pressure, we obtain the wellknown wave equation with a source:

$$
\frac{1}{c^{2}} \frac{\partial^{2} P}{\partial t^{2}}-\frac{\partial^{2} P}{\partial z^{2}}=\frac{\partial \rho}{\partial T} \frac{\partial^{2} T}{\partial t^{2}}
$$

Formula (7) is obtained from (9) if the term with the square of the speed of sound in the denominator is neglected on the left side and the remaining term is double integrated over the coordinate of the heating source on the right side.

In the general case, consideration of the nonlinear response requires solving the complete system (1) - (3). When using the incompressible fluid approximation, an approach is possible 
when the system of continuity and thermal conductivity equations is considered first, and then its solution is used to determine the pressure from the Euler equation (2).

To numerically solve the heat equation, which is necessary when determining the thermoacoustic pressure (7), you can use finite-difference schemes or the Green's function method with subsequent calculation of the corresponding integrals.

$$
\begin{gathered}
T(z, t)=\mathrm{T}_{0}+\int_{0}^{t} \int_{0}^{\infty} G\left(z, z_{1}, t-\tau\right) q_{V}(z, t) \mathrm{d} z_{1} \mathrm{~d} \tau, \\
G\left(z, z_{1}, t\right)=\frac{1}{\sqrt{4 \pi \chi t}}\left\{e^{-\frac{\left(z-z_{1}\right)^{2}}{4 \chi t}}+e^{-\frac{\left(z+z_{1}\right)^{2}}{4 \chi t}}\right\}, \quad \chi=\frac{\kappa}{\rho C_{p}}, \quad q_{V}(z, t)=\frac{Q(z, t)}{\rho C_{p}} \\
\kappa T(z, t)=\int_{-\infty}^{t} \sqrt{\frac{\chi}{\pi(t-\tau)}} q_{s}(\tau) \exp \left\{-\frac{z^{2}}{4 \chi(t-\tau)}\right\} d \tau, \quad \chi=\frac{\kappa}{\rho C_{p}}, \quad q_{s}(t)=I(t)
\end{gathered}
$$

where $\chi$ is the thermal diffusivity.

Formulas (10) and (11) give expressions for the behavior of the temperature profile in the case of bulk and surface absorption, respectively. In the latter case, the formula for surface temperature takes a simple form:

$$
\kappa T(0, t)=\int_{-\infty}^{t} \sqrt{\frac{\chi}{\pi(t-\tau)}} q(\tau) d \tau
$$

\begin{tabular}{|c|c|c|c|c|c|c|}
\hline$T, \mathbf{K}$ & $\begin{array}{l}C_{p}, \mathbf{1 0}^{-3}, \\
\mathrm{~J} /(\mathbf{g} \cdot \mathbf{K})\end{array}$ & $\begin{array}{c}\kappa, \mathbf{1 0}^{-2} \\
\mathrm{~W} /(\mathrm{cm} \cdot \mathrm{K})\end{array}$ & $\begin{array}{c}\chi, \mathbf{1 0}^{-2} \\
\mathrm{~cm}^{2} / \mathrm{s}\end{array}$ & $\begin{array}{c}\text { KTP, } \mathbf{1 0}^{-6}, \\
\mathbf{1} / \mathbf{K}\end{array}$ & $\begin{array}{c}P \\
\text { vap.satur., } \\
\text { bar }\end{array}$ & $\underset{\mathrm{g} / \mathrm{cm}^{3}}{\rho,}$ \\
\hline 300 & 139 & 8 & 4.45 & 181 & $3.68 \cdot 10^{-6}$ & 13.521 \\
\hline 350 & 138 & 8.5 & 4.78 & 182 & $1.23 \cdot 10^{-4}$ & 13.4 \\
\hline 400 & 137 & 9.3 & 5.02 & 182 & $1.61 \cdot 10^{-3}$ & 13.279 \\
\hline 450 & 137 & 9.8 & 5.4 & 183 & 0.01178 & 13.16 \\
\hline 500 & 137 & 10.4 & 6 & 183 & 0.05758 & 13.04 \\
\hline 550 & 137 & 11.5 & 6.48 & 185 & 0.2993 & 12.921 \\
\hline 600 & 137 & 12.4 & 6.84 & 185 & 0.6146 & 12,8 \\
\hline 650 & 137 & 13 & 7.12 & 186 & 1.521 & 12.68 \\
\hline 700 & 137 & 13.5 & 7.41 & 186 & 33 & 12.56 \\
\hline 750 & 137 & 13.8 & 7.67 & 185 & 64.5 & 12.436 \\
\hline 800 & 136 & 14.2 & 7.95 & 185 & 11.58 & 12.31 \\
\hline
\end{tabular}

The results and analysis of specific calculations of the behavior of $P(t)$ are given in the next section for mercury using the known values of its thermophysical parameters [7-9]:

Table 1: Thermophysical parameters of mercury. 


\section{RESULTS AND DISCUSSION.}

\subsection{The dependence of the thermoacoustic signal on the pulse duration.}

First, we consider the change in the thermoacoustic pressure (7) with a decrease in the pulse duration in the target heating from the initial temperature $T_{o}=0.45 T_{b}$ to a predetermined value corresponding to the normal boiling point of mercury $T_{b}=630 \mathrm{~K}$. Because of this additional condition the pressure amplitude increases faster than the reciprocal of the pulse duration, since a shortening of the laser pulse requires an additional increase in its intensity. The considered problem for this reason becomes effectively nonlinear. A similar note also applies to the dependence of the pressure amplitude on the magnitude of the absorption coefficient.

As thermophysical constants in calculating the temperature and pressure, we will use three sets of values from Table 1 corresponding to the target temperatures $T / T_{b}=0.45,1$, and 1.1 .

$$
\begin{gathered}
T_{o}=0.45 T_{b}, \quad \rho=13.59 \frac{\mathrm{g}}{\mathrm{cm}^{3}}, \quad \rho C_{p}=1.865 \frac{\mathrm{J}}{\mathrm{cm}^{3} \mathrm{~K}^{\prime}} \\
\frac{\partial \rho}{\rho \partial T}=1.815 \cdot 10^{-4} \mathrm{~K}^{-1}, \quad \chi=0.032 \frac{\mathrm{cm}}{\mathrm{s}} \\
T_{o}=T_{b}, \quad \rho=12.70 \frac{\mathrm{g}}{\mathrm{cm}^{3}}, \quad \rho C_{p}=1.74 \frac{\mathrm{J}}{\mathrm{cm}^{3} \mathrm{~K}^{\prime}} \\
\frac{\partial \rho}{\rho \partial T}=1.856 \cdot 10^{-4} \mathrm{~K}^{-1}, \quad \chi=0.076 \frac{\mathrm{cm}^{2}}{\mathrm{~s}} \\
T_{o}=1.11 T_{b}, \quad \rho=12.52 \frac{\mathrm{g}}{\mathrm{cm}}, \quad \rho C_{p}=1.716 \frac{\mathrm{J}}{\mathrm{cm}^{3} \mathrm{~K}^{\prime}}, \\
\frac{\partial \rho}{\rho \partial T}=1.86 \cdot 10^{-4} \mathrm{~K}^{-1}, \quad \chi=0.082 \frac{\mathrm{cm}^{2}}{\mathrm{~s}}
\end{gathered}
$$

. Figures 1 and 2 show the behavior of the temperature of the irradiated surface and the thermoacoustic pressure signal in the linear approximation of heating from the initial temperature of $0.45 T_{b}$ to $T_{b}$ at various durations of the absorbed laser pulse with a decrease from 30 to $0.1 \mathrm{~ns}$. The thermophysical parameters indicated in (13) - (15) were used at $T_{0} / T_{b}$ $=0.45$ and 1 and two absorption coefficients of $10^{5} \mathrm{~cm}^{-1}$ and $10^{6} \mathrm{~cm}^{-1}$.

From a comparison of Figures 1 and 2, it can be seen that the transition from the use of data (13) to data (14) practically does not affect the shape of the pressure response, with the exception of the ratio between the amplitudes of the positive and negative pressure waves, the values of which approach unity with decreasing pulse duration and absorption coefficient. This behavior is associated with a relative change in the length of the thermal effect due to thermal conductivity and the radiation absorption length .

However, the amplitude values of pressure and absorbed intensity change more significantly with a change in the pulse duration, as is also shown in Table 2, where these values are given for a set of constants (13) and (14) for two absorption coefficients of $10^{5} \mathrm{~cm}^{-1}$ and $10^{6} \mathrm{~cm}^{-1}$. 

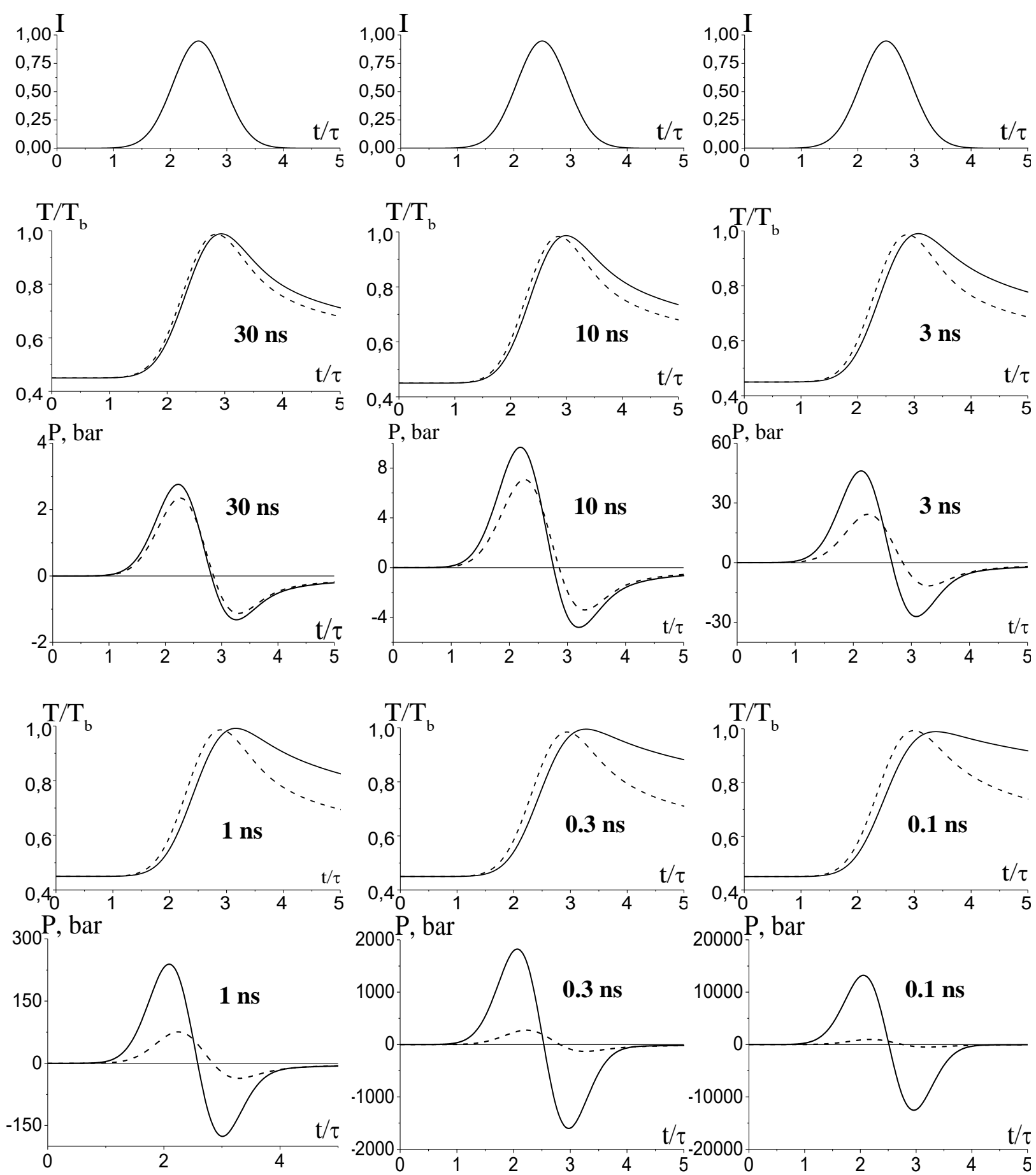

Fig. 1 Temporal profile of intensity $(I)$, relative temperature $\left(T / T_{b}\right)$ and pressure $(P)$ for different pulse durations (they are shown in the graphs) and $\alpha=10^{5} \mathrm{~cm}^{-1}$ (solid lines), $\alpha=10^{6} \mathrm{~cm}^{-1}$ (dashed lines). The values of maximum intensities for two absorbtion cooficients and difeerent pulse duration are: $30 \mathrm{~ns}-\mathrm{I}_{\mathrm{m}}=1.2 \mathrm{MW} / \mathrm{cm}^{2}$ and $\mathrm{I}_{\mathrm{m}}=1.04 \mathrm{MW} / \mathrm{cm}^{2} ; 10 \mathrm{~ns}-\mathrm{I}_{\mathrm{m}}=2.3 \mathrm{MW} / \mathrm{cm}^{2}$ and $\mathrm{I}_{\mathrm{m}}=1.8 \mathrm{MW} / \mathrm{cm}^{2} ; 3 \mathrm{~ns}$

- $\mathrm{I}_{\mathrm{m}}=5 \mathrm{MW} / \mathrm{cm}^{2}$ and $\mathrm{I}_{\mathrm{m}}=3.4 \mathrm{MW} / \mathrm{cm}^{2} ; 1 \mathrm{~ns}-\mathrm{I}_{\mathrm{m}}=11 \mathrm{MW} / \mathrm{cm}^{2}$ and $\mathrm{I}_{\mathrm{m}}=6 \mathrm{MW} / \mathrm{cm}^{2} ; 0.3 \mathrm{~ns}-\mathrm{I}_{\mathrm{m}}=29$

$\mathrm{MW} / \mathrm{cm}^{2}$ and $\mathrm{I}_{\mathrm{m}}=12 \mathrm{MW} / \mathrm{cm}^{2} ; 0.1 \mathrm{~ns}-\mathrm{I}_{\mathrm{m}}=74 \mathrm{MW} / \mathrm{cm}^{2}$ and $\mathrm{I}_{\mathrm{m}}=23.3 \mathrm{MW} / \mathrm{cm}^{2}$. 

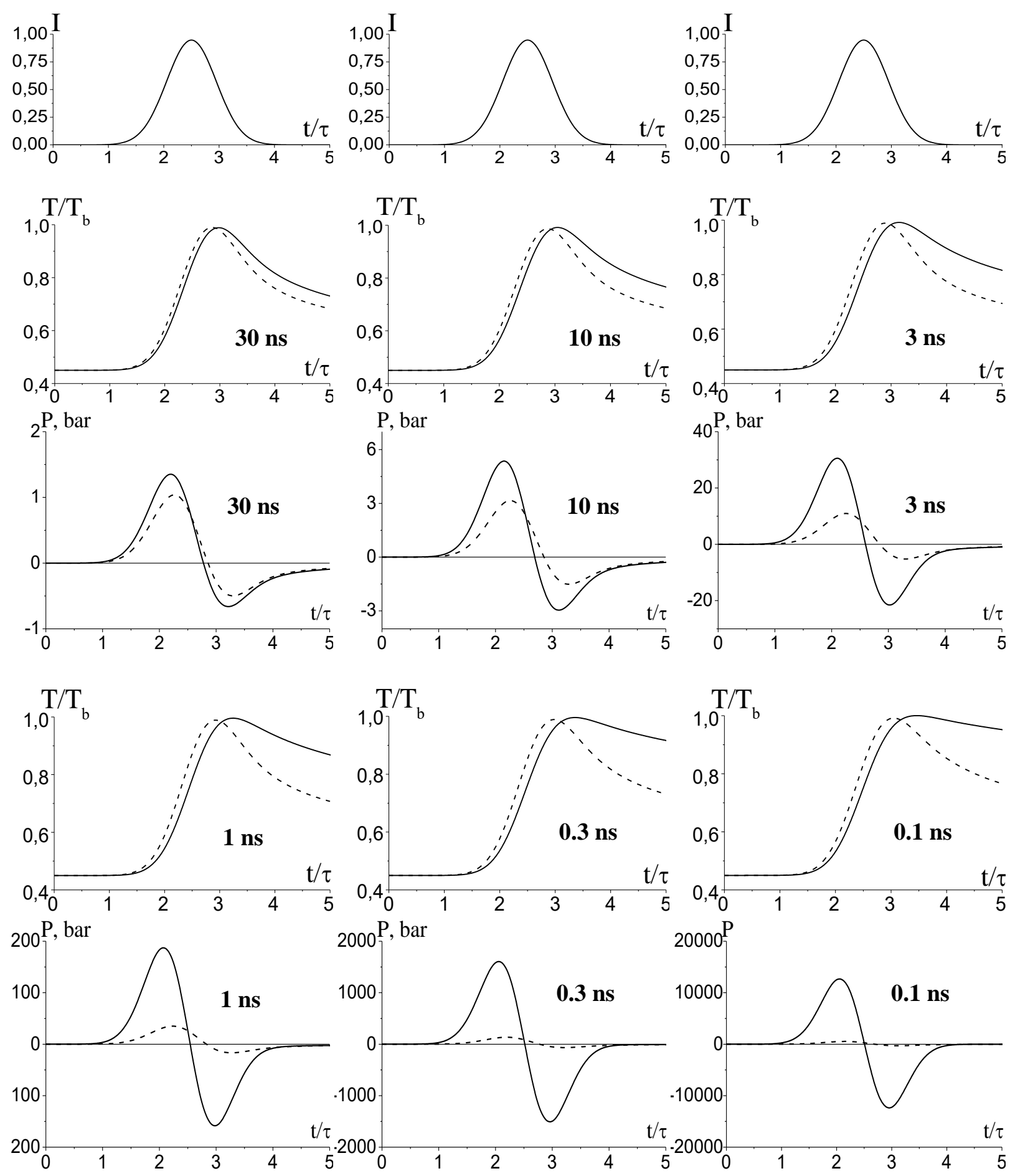

Fig. 2 Temporal profile of intensity $(I)$, relative temperature $\left(T / T_{b}\right)$ and pressure $(P)$ for different pulse durations (they are shown in the graphs) and $\alpha=10^{5} \mathrm{~cm}^{-1}$ (solid lines), $\alpha=10^{6} \mathrm{~cm}^{-1}$ (dashed lines). The values of maximum intensities for two absorbtion cooficients and difeerent pulse duration are: $30 \mathrm{~ns}-\mathrm{I}_{\mathrm{m}}=1 \mathrm{MW} / \mathrm{cm}^{2}$ and $\mathrm{I}_{\mathrm{m}}=0.7 \mathrm{MW} / \mathrm{cm}^{2} ; 10 \mathrm{~ns}-\mathrm{I}_{\mathrm{m}}=1.8 \mathrm{MW} / \mathrm{cm}^{2}$ and $\mathrm{I}_{\mathrm{m}}=1.3 \mathrm{MW} / \mathrm{cm}^{2} ; 3 \mathrm{~ns}-$

$\mathrm{I}_{\mathrm{m}}=4.2 \mathrm{MW} / \mathrm{cm}^{2}$ and $\mathrm{I}_{\mathrm{m}}=2.4 \mathrm{MW} / \mathrm{cm}^{2} ; 1 \mathrm{~ns}-\mathrm{I}_{\mathrm{m}}=10 \mathrm{MW} / \mathrm{cm}^{2}$ and $\mathrm{I}_{\mathrm{m}}=4.5 \mathrm{MW} / \mathrm{cm}^{2} ; 0.3 \mathrm{~ns}-\mathrm{I}_{\mathrm{m}}=28$ $\mathrm{MW} / \mathrm{cm}^{2}$ and $\mathrm{I}_{\mathrm{m}}=12 \mathrm{MW} / \mathrm{cm}^{2} ; 0.1 \mathrm{~ns}-\mathrm{I}_{\mathrm{m}}=74 \mathrm{MW} / \mathrm{cm}^{2}$ and $\mathrm{I}_{\mathrm{m}}=18 \mathrm{MW} / \mathrm{cm}^{2}$. 


\begin{tabular}{|c|c|c|c|c|c|c|c|c|}
\hline & \multicolumn{5}{|c|}{$\alpha=10^{5} \mathrm{~cm}^{-1}$} & \multicolumn{4}{|c|}{$\alpha=10^{6} \mathrm{~cm}^{-1}$} \\
\hline $\begin{array}{c}\tau_{\mathrm{p} .,} \\
\mathrm{ns}\end{array}$ & $\begin{array}{c}\mathrm{I}_{\mathrm{m} 1,} \\
\mathrm{MW} / \mathrm{cm}^{2}\end{array}$ & $\begin{array}{c}\mathrm{P}_{\mathrm{m} 1,} \\
\mathrm{bar}\end{array}$ & $\begin{array}{c}\mathrm{I}_{\mathrm{m} 2,} \\
\mathrm{MW} / \mathrm{cm}^{2}\end{array}$ & $\begin{array}{c}\mathrm{P}_{\mathrm{m} 2,} \\
\text { bar }\end{array}$ & $\begin{array}{c}\mathrm{I}_{\mathrm{m} 1,} \\
\mathrm{MW} / \mathrm{cm}^{2}\end{array}$ & $\begin{array}{c}\mathrm{P}_{\mathrm{m} 1,} \\
\mathrm{bar}\end{array}$ & $\begin{array}{c}\mathrm{I}_{\mathrm{m} 2,} \\
\mathrm{MW} / \mathrm{cm}^{2}\end{array}$ & $\begin{array}{c}\mathrm{P}_{\mathrm{m} 2,} \\
\text { bar }\end{array}$ \\
\hline 0,1 & 74 & 140000 & 74 & 140000 & 18.2 & 600 & 23.3 & 1100 \\
\hline 0,3 & 27.5 & 1750 & 29.2 & 2000 & 9 & 150 & 11.9 & 300 \\
\hline 1 & 10 & 210 & 11.2 & 260 & 4.45 & 38 & 6.1 & 80 \\
\hline 3 & 4.24 & 33 & 5.07 & 50 & 2.43 & 12.5 & 3.4 & 27 \\
\hline 10 & 1.82 & 5.75 & 2.3 & 10 & 1.28 & 3.5 & 1.81 & 7.6 \\
\hline 30 & 0.9 & 1.45 & 1.2 & 3 & 0.73 & 1.15 & 1.04 & 2.6 \\
\hline
\end{tabular}

Table 2: The values of the maximum pressure and the corresponding radiation intensity depending on the duration of the absorbed laser pulse for two values of the absorption coefficient and heating to $T_{b}$. from initial temperature $T_{o}=0.45 T_{b}$.

The ratio of the pressure amplitudes calculated for different sets of constants (13), (14) at various values of the absorption coefficient is shown in Fig. 3 (two upper curves) depending on the duration of the laser pulse. The solid curve $\left(\alpha=10^{5} \mathrm{~cm}^{-1}\right)$ tends to unity for short pulses where the heat conduction effect is smaller than at $\alpha=10^{6} \mathrm{~cm}^{-1}$.

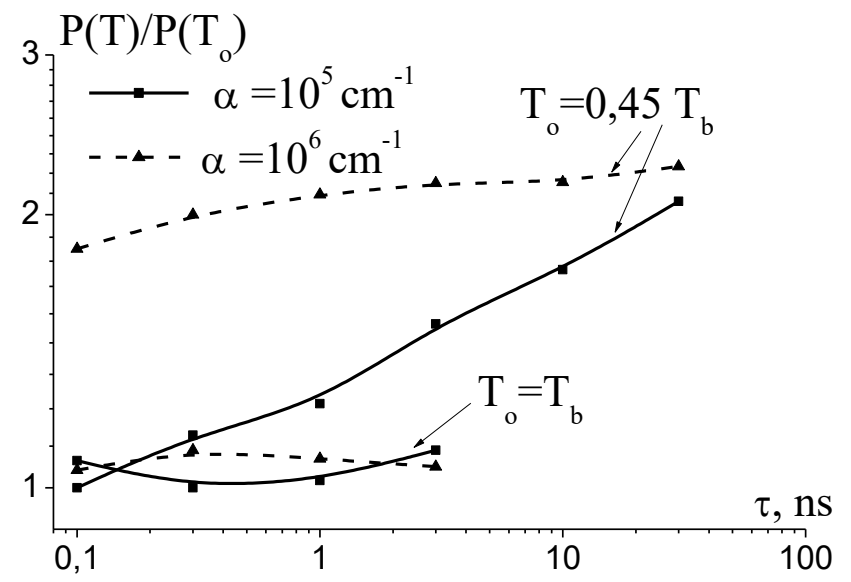

Fig. 3 Ratio of maximum pressures at various thermophysical parameters for two values of the absorption coefficient depending on the pulse duration.

The pressure in Fig. 1 and Fig. 2 for $30 \mathrm{~ns}$ is approximately consistent with the calculation result in [1]. It also follows from the figures and Table 2 that the amplitude of the thermoacoustic pressure signal grows much faster than $\left(\tau_{p}\right)^{-1}$ when $\tau_{p}$ diminishes. This difference, clearly demonstrated in Fig. 3, as mentioned above, is associated with an additional condition for reaching a given temperature $T_{o}=T_{b}$ for each pulse duration.

Using simple estimates, it is easy to find that in this case, with the volume or surface nature of the absorption, the amplitude of the linear response with decreasing pulse duration will increase as $\left(\tau_{p}\right)^{-2}$ or $\left(\tau_{p}\right)^{-3 / 2}$. With a comparable effect from these two heating mechanisms, the exponent will lie in the range $1.5-2$, as can be seen from Fig. 4.

Due to such a rapid increase, the amplitude of the thermoacoustic signal exceeds the atmospheric pressure by more than an order of magnitude even with a duration of $3 \mathrm{~ns}$. Such an excess, which increases with a further decrease in the pulse duration, greatly complicates 
the direct application of the method used in [1] for calibrating a piezoelectric sensor in the case of short laser pulses.

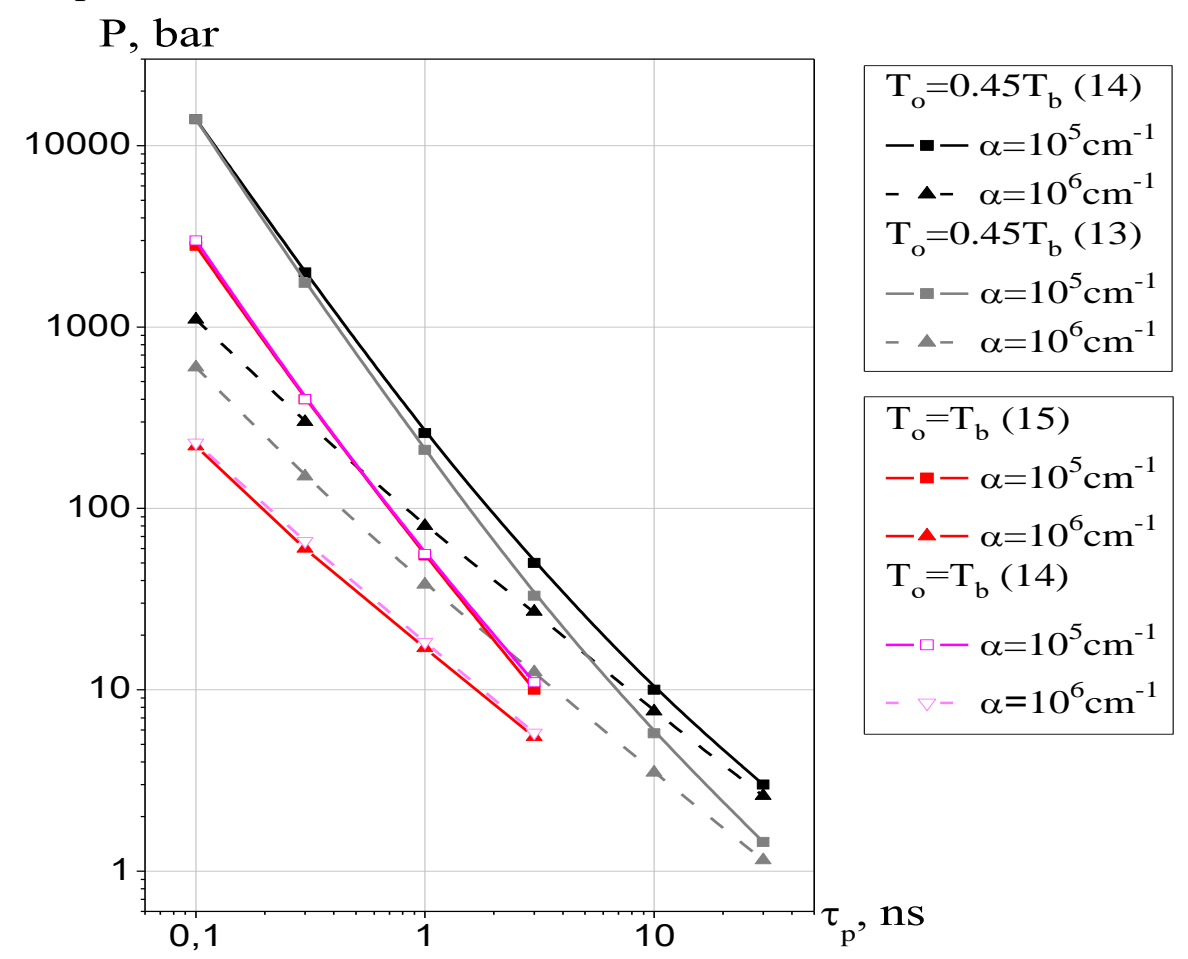

Fig. 4 Change in pressure maximum depending on the pulse duration for two sets of thermophysical constants (13)-(14) (grey and black) with initial temperature $T_{o}=0.45 T_{b}$ and (14)-(15) (violet and red) with initial temperature $T_{o}=T_{b}$. Triangles and squares correspond to two values of the absorption coefficient $\alpha=10^{6} \mathrm{~cm}^{-1}$ and $\alpha=10^{5} \mathrm{~cm}^{-1}$, respectively.

\subsection{Modification of the approach for short pulses.}

A decrease in pressure for short pulses can be achieved by preheating the target to a temperature close to the value of $T_{b}$ with an additional longer laser pulse. The two-pulse technique, when the first pulse transfers the irradiated system to the desired state, and the second carries out its monitoring is widely used in various situations (see, for example, [10]). In our case, the short pulse should be combined with the moment of reaching the temperature maximum from the first long pulse, when the thermoacoustic signal generated by it is close to its passage through zero.

The action of a short laser pulse should provide heating of the target from $T_{b}$ to values at which the saturated vapor pressure exceeds 3 bar. For mercury, in accordance with Table 1 (saturation pressure versus temperature), this gives $T$ value about $1.1 T_{b}$ The ablation regime then approaches evaporation into vacuum with a recoil pressure of $P_{r} \cong 1$ bar. However, a consistent quantitative analysis of the problem of the unsteady process of formation of a pulse of evaporative pressure under similar conditions, when the saturated vapor pressure as a result exceeds the external pressure by several times, as far as we know, has not yet been carried out. Note that in the conditions of interest to us, the evaporation and thermoacoustic signals are simply summed, since the cooling effect of evaporation on the temperature profile is small. 
Figure 5 shows the temporal dependences of the thermoacoustic pressure upon heating the target from $T_{0}=T_{b}$ to $T=1.1 T_{b}$ with laser pulses of various durations $(3,1,0.3$, and 0.1 $\mathrm{ns}$ ), and Table 3 shows the maximum achievable values of thermoacoustic pressure under such conditions.

It can be seen that the magnitude of the thermoacoustic signal is much smaller than in Fig. 1 and Table 2; however, at $0.1 \mathrm{~ns}$ its amplitude nevertheless considerably exceeds 1 bar even for the absorption coefficient $10^{6} \mathrm{~cm}^{-1}$. The total thermoacoustic signal when exposed to two pulses with durations of $30 \mathrm{~ns}$ and $1 \mathrm{~ns}$ is shown in Fig. 6.
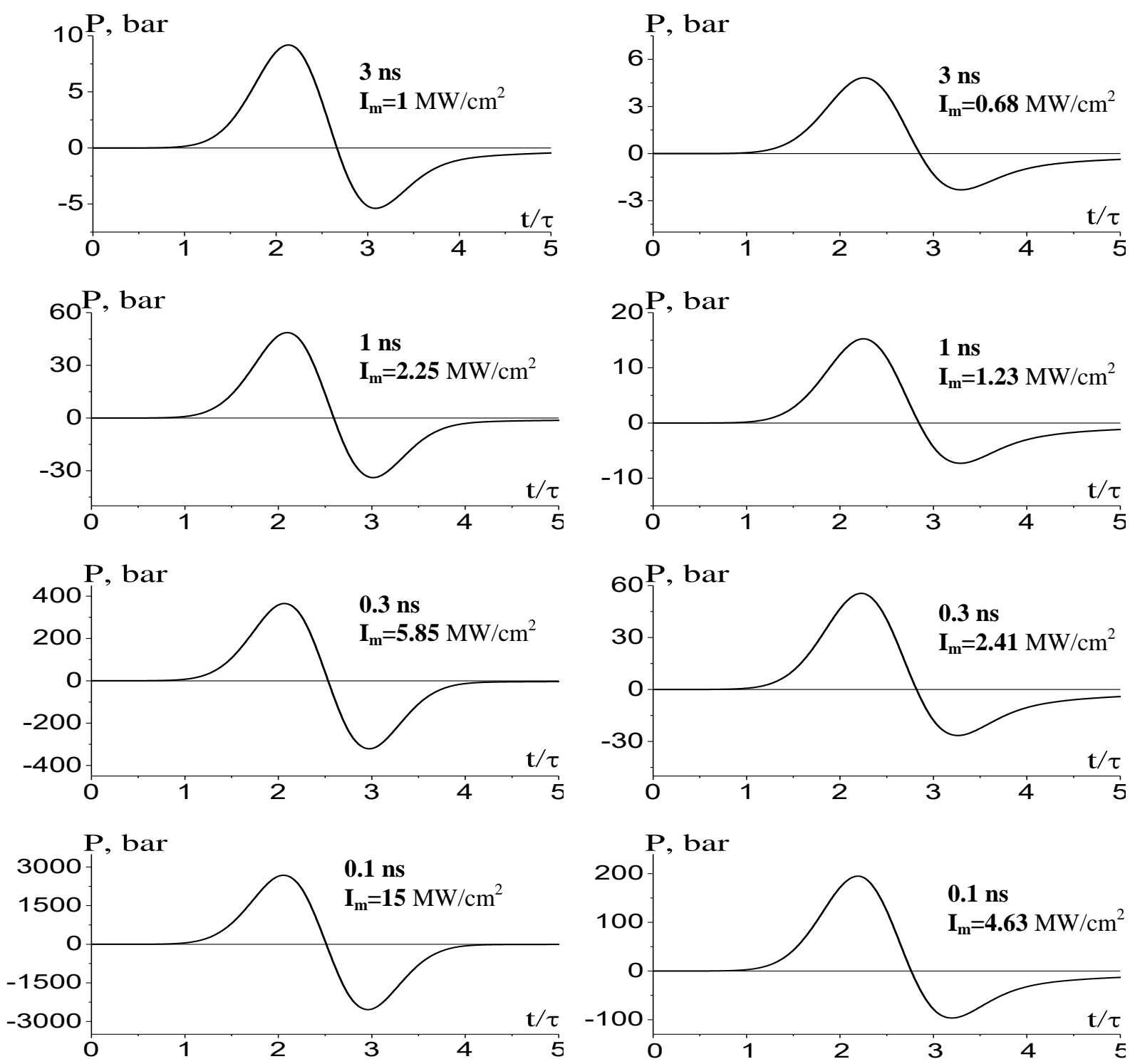

a

b

Fig.5. Temporal profile of thermoacoustic pressure depending on the laser pulse duration and $\alpha=10^{5}$ $\mathrm{cm}^{-1}(\mathbf{a}), \alpha=10^{6} \mathrm{~cm}^{-1}(\mathbf{b})$ upon heating of the target from $T=T_{b}$ to $T=1.11 T_{b}$ with the corresponding intensities. 


\begin{tabular}{|c|c|c|c|c|c|c|c|c|}
\hline & \multicolumn{4}{|c|}{$\alpha=10^{5} \mathrm{~cm}^{-1}$} & \multicolumn{4}{|c|}{$\alpha=10^{6} \mathrm{~cm}^{-1}$} \\
\hline$\tau, \mathrm{HC}$ & $\begin{array}{c}\mathrm{I}_{\mathrm{m} 1}, \\
\mathrm{MW} / \mathrm{cm}^{2}\end{array}$ & $\begin{array}{l}\mathrm{P}_{\mathrm{m} 1} \\
\text { bar }\end{array}$ & $\begin{array}{c}\mathrm{I}_{\mathrm{m} 2} \\
\mathrm{MW} / \mathrm{cm}^{2}\end{array}$ & $\begin{array}{l}\mathrm{P}_{\mathrm{m} 2} \\
\text { bar }\end{array}$ & $\begin{array}{c}\mathrm{I}_{\mathrm{m} 1} \\
\mathrm{MW} / \mathrm{cm}^{2}\end{array}$ & $\begin{array}{l}\mathrm{P}_{\mathrm{m} 1}, \\
\text { bar }\end{array}$ & $\begin{array}{c}\mathrm{I}_{\mathrm{m} 2}, \\
\mathrm{MW} / \mathrm{cm}^{2}\end{array}$ & $\begin{array}{l}\mathrm{P}_{\mathrm{m} 2,} \\
\text { bar }\end{array}$ \\
\hline 0.1 & 15 & 2800 & 15 & 3000 & 4.63 & 220 & 4.73 & 230 \\
\hline 0.3 & 5.85 & 400 & 5.87 & 400 & 2.41 & 60 & 2.47 & 66 \\
\hline 1 & 2.25 & 55 & 2,27 & 56 & 1.23 & 17 & 1.25 & 18.3 \\
\hline 3 & 1.01 & 10 & 1.03 & 11 & 0.675 & 5.5 & 0.7 & 5.8 \\
\hline
\end{tabular}

Table 3: The values of the maximum pressure and the corresponding radiation intensity depending on the duration of the absorbed laser pulse for two values of the absorption coefficient and heating up to $1.11 T_{b}$, from the initial temperature $T_{o}=T_{b}$.

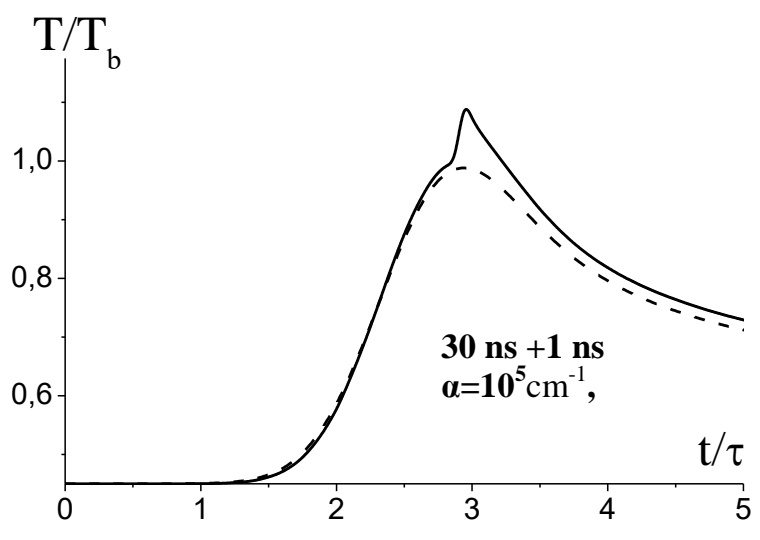

$\mathbf{a}$

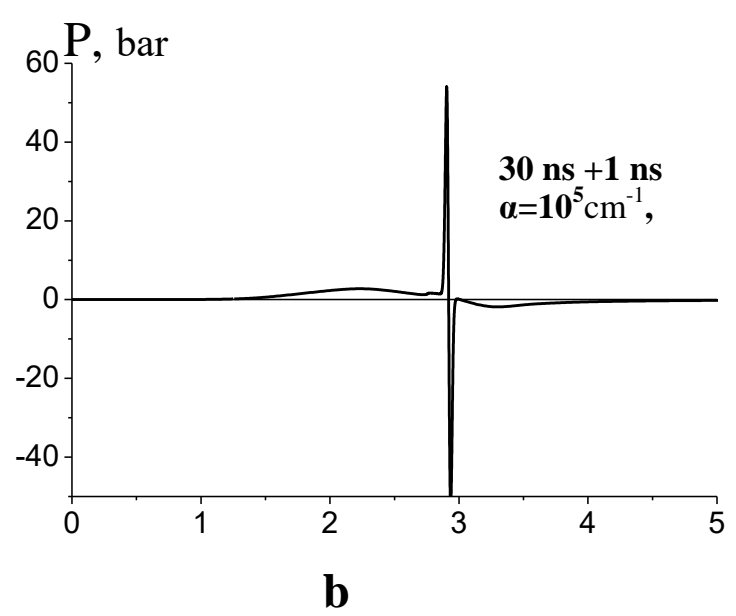

b

Fig. 6. The temporal profile of temperature (a) and pressure (b) for a laser pulse with a duration of 30 ns $I_{m l}=1.2 \mathrm{MW} / \mathrm{cm}^{2}$ followed by heating of the target from $T=T_{b}$. up to $T=1.11 T_{b} .1 \mathrm{~ns}$ pulse with

$$
I_{m 2}=3.2 \mathrm{MW} / \mathrm{cm}^{2} ; \alpha=10^{5} \mathrm{~cm}^{-1} \text {. }
$$

Note that for $\alpha=10^{6} \mathrm{~cm}^{-1}$ and a pulse duration of $0.3 \mathrm{~ns}$, the maximum of the acoustic pressure is only slightly higher than the maximum for the duration of 1 ns, shown in Fig. 6 This means that calibration in the case under consideration is possible for the subnanosecond mode if the absorption coefficient is large enough.

The question of choosing the wavelength of laser radiation to ensure such a value of $\alpha$ is beyond the scope of this paper. At the same time, for mercury at higher temperatures approaching the critical point, there appears to be a sharp decrease in $\alpha$, characteristic of the metal-insulator transition (see, for example, [11-14] and referencies therein).

\subsection{Applicability of the approximations used.}

The use of the equation of state in this work, which takes into account the dependence of the density of the liquid only on temperature, suggests, in particular, the slowness of the processes under consideration on a scale specified by the speed of sound $c_{s}$. This means that the convective velocity $v$ associated with thermal expansion should be small compared to $c_{s}$ $>>v$ and the characteristic spatial size 1 of the processes considered over time $t$ should be small relative to the mean free path of sound over the same time. This inequality holds in all of the exposure modes discussed. 
The effect of the calculated pressure $P$ on the density change can be approximately estimated using a simple relation that takes into account the proportionality of this change to the ratio $P / c_{s}^{2}$. This contribution is significant only for the shortest pulses during direct target heating from the initial room temperature to the boiling point. However, these cases are not of interest for calibration precisely because of the large magnitude of the resulting thermoacoustic pressure.

The applicability of the linear approximation is evidenced by a comparison of the results obtained when two sets of constants corresponding to the initial and final values of the target temperature are used in the calculations. The results are shown in Fig. 3 and in Tables 2 and 3. For the heating interval $T=(0.45-1) T_{b}$, the difference between the sets (13) and (14) is not negligible due to a noticeable variation in thermal diffusivity in this range. However, this difference does not play a significant role, if we take into account the use of a two-pulse technique. At the same time, for short pulses with a heating interval $T=(1-1.1) T_{b}$, the applicability of the linear approximation is quite satisfactory, as can be seen from Fig. 3 (two lower curves), table 3 and Fig. 4 where in the case $T_{o}=T_{b}$ there is no visible difference between the curves with the sets (14) and (15) for the same $\alpha$ value.

\section{CONCLUSION.}

Thus, in the present work, as a result of numerical simulation of various modes of the method of calibrating pressure sensors, originally proposed and implemented [1] for a laser pulse duration of $30 \mathrm{~ns}$, it is shown that the direct extension of this method to shorter durations is difficult. This is due to the rapid increase in thermoacoustic pressure with decreasing pulse duration, which turns out to be sharper than the inverse dependence characteristic of a linear response to a given laser intensity, due to the fixation of the temperature range in the calibration mode

A natural way to overcome this difficulty is to use a double-pulse heating of the target, when the first long pulse brings the temperature of the target closer to the boiling point, and the second, shorter one, provides the appearance of an evaporative signal with a reduced (compared to single-pulse mode) value of the thermoacoustic signal. This approach allows us to extend the calibration method implemented in [1] to subnanosecond pulses, thereby expanding its range of applicability by almost two orders of magnitude in reducing the laser pulse duration.

\section{REFERENCES.}

[1] A.A.Samokhin , E.V.Shashkov, N.S., Vorobiev, A.E Zubko., "Nanosecond Calibration of a Piezo Transducer by Comparing Thermoacoustic and Vaporization Pressure Signals at Pulsed Laser Irradiation of a Metal Target", Physics of Wave Phenomena, 27 (4), 268-270 (2019)

[2] M.W. Sigrist and F.K. Kneubuhl, "Laser-generated stress waves in liquids", Journal Acoustical Society of America, 64(6), 1652-1663 (1978).

[3] A.A.Samokhin "First-order phase transitions induced by laser radiation in absorbing condensed matter", Proceedings of the Institute of General Physics Academy of Science of the USSR, Commack, New York, 13, 1-161, (1990).

[4] I.A. Veselovskii, B.M. Zhiryakov, N.I. Popov, A.A. Samokhin, "'The photoacoustic effect andphase transitions in semiconductors and metals irradiated by laser pulses", Proceeding of the Institute of General Physics Academy of Sciences of the USSR, 13, 179-198 (1990). 
[5] A.E. Zubko, A.A. Samokhin, "Modeling of thermoacoustic and evaporation pressure signals in absorbing liquids irradiated with nanosecond laser pulses", Mathematica Montesnigri, 36,78-85 (2016).

[6] A.E.Graham, F.W.Neilson, W.B.Benedick, "Piezoelectric current from schock-loaded quartz - a submicrosecond stress gauge", J. Appl. Phys., 36 (5), 1775-1783 (1965)

[7] V.E. Zinov'ev. Teplofizitcheskie svoistva metallov pri visokich temperaturach, Moskva: Metallurgiya (1989).

[8] A.P. Babichev, N.A. Babushkina, A.M. Bratkovskiy, Fizitcheskie velitchini, Moskva: Energoatomizdat (1991).

[9] A.I. Volkov, I.M. Jarskiy, Bol'shoy chimitcheskiy spravotchnik, Moskva: Sovetskaya shkola (2005).

[10] A.A. Samokhin, S.M. Klimentov, P.A. Pivovarov, "Acoustic diagnostics of the explosive boiling up of a transparent liquid on an absorbing substrate induced by two nanosecond laser pulses", Quantum Electron., 37 (10), 967-970 (2007).

[11] S.N. Andreev, V.I. Mazhukin, N.M. Nikiforova, A.A. Samokhin, "On possible manifestations of the induced transparency during laser evaporation of metals", Quantum Electronics, 33, 771776 (2003).

[12] A.A. Samokhin, A.E. Zubko, "On metal-dielectric transition in laser ablation modeling", Mathematica Montesnigri, 46, 114-122 (2019).

[13] Yu Zhang, Daixian Zhang, Jianjun Wu, Zhen He, and Xiong Deng, "A thermal model for nanosecond pulsed laser ablation of aluminum", AIP Advances, 7, 075010 (2017)

[14] A. A..Samokhin, E.V..Shashkov, N. S..Vorobiev, A.E..Zubko, "On acoustical registration of irradiated surface displacement during nanosecond laser-metal interaction and metal-nonmetal transition effect", Appl. Surf. Science, 502, 144261 (2020).

Received May 10, 2020 